\title{
Experience of 4 Cases of Treatment of Gynecomastia from Dialectical Analysis of Liver and Kidney
}

\author{
Xiaohua Liu*, Guangshen Bai \\ Beijing Miyun District Traditional Chinese Medicine Hospital, Beijing 101500, China \\ Email:22251316@qq.com
}

\begin{abstract}
The author adopts the method of syndrome differentiation and treatment in Chinese medicine based on the treatment of 4 cases of gynecomastia from dialectical analysis of liver and kidney. The report is as follows.

Keywords: gynecomastia, dialectical treatment of liver and kidney
\end{abstract}

Gynecomastia is a condition in which one or both breasts develop like women and become enlarged. It usually occurs in young adults or adults. Patients often develop one or both breast hypertrophy or areolar footwall mass. At first, it often occurs below the areola, and the mass is rubbery and with unclear boundary. At times, people can feel tenderness or pain with little chances of malignant change on most occasions ${ }^{[1]}$. In recent years, the incidence and diagnosis rate of gynecomastia have increased significantly due to the changes in dietary structure and life pressure and other factors, which can cause physical discomfort and emotional tension in patients. And people can be confused in distinguishing this disease and other breast diseases, especially breast cancer ${ }^{[2]}$. Modern medicine for the treatment of this disease is mostly offered to supplement androgen or inhibit the activity of estrogen receptor methods, which has a great side effect. The treatment method of traditional Chinese medicine highlights the advantage. Gynecomastia belongs to areolar nodule in traditional Chinese medicine. The name of areolar nodule comes from A Complete Book on the Experience of Sores and Ulcers, and is also called "breast nodule" ${ }^{[3]}$. The author is a western medicine surgeon. After systematically learning the relevant knowledge of traditional Chinese medicine, especially the breast surgery of traditional Chinese medicine, the author adheres to the treatment of gynecomastia based on the syndrome differentiation of liver and kidney, and has achieved certain results. Here are 4 cases to illustrate.

\section{Case data}

\subsection{Case 1}

A 41-year-old male patient had developed breast pain for more than 1 month. The patient came to our hospital 1 month ago with emotional fluctuations, and later realized that the left breast was larger than before, with swelling, pain, obvious tenderness and irritability. Physical examination: bilateral mammary glands were symmetrical, glandular tissue was palpable under the left nipple, about $2 * 2 \mathrm{~cm}$, with clear boundary, tough quality and obvious tenderness. Symptoms showed that the tongue was red, the coating on the tongue was yellow, and the pulse was wiry. B-ultrasonography revealed a hypoechoic area below the left nipple (gland development).

\subsubsection{The initial diagnosis and treatment}

Dialectical analysis showed stagnation of the circulation of vital energy and liver stagnation, treatment was conducted to soothe the liver, regulate Qi and relieve pain.

Prescription: Bupleurum 12g; Scutellaria 12g; Peony 9g; Fried Gardenia 12g; Green peel 6g; Tangerine peel 6g; Fragrant 9g; Prunella 15g; Rough oyster 20g; Seaweed 12g; Poria cocos 15g; Pinellia chinensis 6g; Yanhuso 9g; Frankincense 6g; Myrrh 6g; Fructus Aurantii 9g; Rhubarb 3g

Take one dose a day in morning and evening (7 dosages).

\subsubsection{The further consultation}

After administering 7 doses of medication, the pain in the left breast was less than before. Hyperplastic glands were still palpable. Symptoms showed that the tongue was red, the coating on the tongue was yellow, and the pulse was wiry. Dialectical analysis demonstrated that the depression of the liver generates pathogenic fire, and treatment can be conducted to soothe liver and clear fire, dissolve phlegm and disperse knot.

Prescription: Fructus Aurantii 9g; Bupleurum 12g; Scutellaria Radix 12g; Green Tangerine Peel 6g; Tree Peony Bark 9g; Incense 9g; Prunella 15g; Raw oyster 20g; Poria cocos 15g; Pinellia pinellia 6g; Yuanhu 9g; Sanli 12g; Curcuma 
zedoary 12g; Angelica 12g; Antler cream 15g; cistanche 15g; Xianling spleen 9g; Xianling spleen 12g; Rose 6g

Take one dose a day in morning and evening (7 dosages).

\subsubsection{The third diagnosis}

The pain of the left breast was significantly relieved. A induration, about $1 * 1 \mathrm{~cm}$ in size, could be felt at 8 o'clock in the left nipple. The tongue was red, the coating of the tongue was yellow and greasy, and the pulse was wiry. Dialectical analysis showed stagnation of the circulation of vital energy and liver stagnation, kidney empty and cuspidor.

Prescription: Peony bark peeled with roasted Rhizoma Atractylodis Macrocephalae15g; Roasted rhizoma atractylodis $15 \mathrm{~g}$

\subsubsection{The fourth diagnosis}

Symptoms significantly reduced, and no obvious discomfort showed in the left breast. Symptom of mild pharyngeal itching and dry pharynx, red tongue, thin white coating, wiry pulse were shown.

Prescription: Fragrance 9g; Prunella 15g; Raw oyster 20g; Fructus Aurantii 9g; Atractylodes macrocephala 15g; Bupleurum 12g; Scutellaria baicalensis 12g; Qingpi 6g; Tangerine peel 6g; Mint 9g; Poria cocos 15g; Yanhuso 9g; Triangulum 12g; Zedoary 12g; Radix Rhizoma 6g; Angelica 12g; Antler Cream 15g; Cistanche 15g; Rose 6g

\subsubsection{The fifth diagnosis}

The symptom of pain was completely relieved and the left breast was soft without obvious induration. Stop administering medicines

\subsection{Case 2}

A 49-year-old male patient presented parochial pain in the left nipple for one month. The patient reported paroxysmal pain in the left nipple for nearly 1 month, which was obvious when touched the wearing clothes, which was accompanied by dysphoria in chestpalms-soles, less sleep and more dreams at night. He was diagnosed as "hyperplasia of mammary glands" in another hospital, and he was given Xiaojin capsule for oral administration, but the effect was not good, so he came the hospital for further diagnosis. He had a history of hypertension for 5 years and diabetes for 2 years, and the indicators of control of chronic diseases by oral drugs reported by himself were satisfactory. Examination: bilateral breast asymmetry, bulge of left breast, palpable gland tissue, about $4 * 4 \mathrm{~cm}$, gentle tenderness, clear boundary, good mobility, nipple without depression, nipple extrusion without abnormal secretions. Symptoms showed that the tongue was red, the coating on the tongue was yellow, and the pulse was wiry. B-ultrasonography revealed glandular echo (breast development) below the left nipple.

\subsubsection{The initial diagnosis}

Dialectical analysis showed the Liver-Kidney Yin deficiency, Qi stagnation and spittoon throat syndrome, treatment can be made to soothe the liver and regulate Qi, to soften hardness and dissipate stagnation.

Prescription: Bupleurum 9g; Scutellaria 9g; Peony bark 9g; Gardenia 9g; Green peel 6g; Tangerine peel 6g; trichosanthin 15g; Incense 9g; Fructus trichosanthis 15g; Aurantii 6g; Dandelion 15g; Rhubarb 3g; Seaweed 9g; Yanhuso 9g; Raw oyster $20 \mathrm{~g}$

Take one dose a day in morning and evening (7 dosages).

\subsubsection{The further consultation}

After taking 7 doses, breast pain relieved, but there is still pain, when the touch is obvious. Dark ecchymosis on the tongue, thin white moss, pulse and string, thyroid function examination is normal. Dialectical for deficiency of liver and kidney Yin, qi stagnation and cuspidor, treatment to nourish Yin phlegm, soft and firm sankie.

Prescription: Rehmannia rehmanniae 30g; Dogwood meat 30g; Antler cream 15g; Dodder 15g; Wine desert cistanche 15g; Peony peel 9g; Fried gardenia 9g; Tangerine peel 6g; Yanhuso 9g; Raw oyster 20g; Raw keel 20g; Trichosanthin 15g; Chuanxiong 9g; Lily $15 \mathrm{~g}$

14 doses in total, and administer one dose a day in morning and evening separately.

\subsubsection{The third diagnosis}

Symptoms improved significantly, and the left breast was only occasionally painful when touched.

Roasted Gardenia and Chaihuum 9g; Angelica 9g; White peony 9g; Licorice 3g; Turmeric 9g; Dandelion 9g

\subsubsection{The fourth diagnosis}

There was no obvious discomfort symptoms. There was occasional left mammary gland discomfort after touched. Symptoms showed that the tongue was red, the coating on the tongue was yellow, and the pulse was wiry. At present, there was no recurrence after six months of follow-up. 


\subsection{Case 3}

The patient was male, 64 years old, with slow swelling and enlargement of the left breast for four months, accompanied by paroxysmic pain. The pain was obvious when touching the clothes, accompanied by soft and sour waist and knee, fear of cold, warm hands and feet, and poor sleep at night. It was diagnosed as "male breast development disease" by another hospital. Xiaojin capsule was taken orally for three months, the effect was not good, and the drug was stopped by itself.In the past month, the breast enlargement was obvious, so I came to our hospital for treatment.She had a history of diabetes for 10 years and took medication to control her blood sugar steadily. Physical examination: bilateral breast asymmetry, left breast bulge, normal skin color, left breast central agglomeration, tough, about $4 * 4 \mathrm{~cm}$ in size, high tension, tenderness, pale tongue, white fur, heavy pulse.

\subsubsection{The initial diagnosis}

Dialectical diagnosis showed kidney Yang deficiency and failure. Treatment can be made to warm and tonify kidney Yang, relieve liver and disperse knots.

Prescription: Radix rehmanniae recen 15g; Chinese yam 15g; Common macrocarpium Fruit 15g; Alisma alisma 12g; Herba Epimedii 15g; Root-bark of tree peony 12g; Fritillaria chuanensis 12g; White peony root 15g; Radix Aucklandiae $15 \mathrm{~g}$; Turmeric $15 \mathrm{~g}$.

Take one dose a day in morning and evening (7 dosages).

\subsubsection{The further consultation}

After taking the medicine, the texture of the left breast became softer, but there was still pain. Sleep was improved, and the mood was more peaceful than before. Add a soft and solid drug to the front center.

Prescription: Chinese honeylocust spine $15 \mathrm{~g}$; Common macrocarpium fruit 15g; Radix Aucklandiae 12g; radix curcumae $18 \mathrm{~g}$

14 doses in total, and administer one dose a day in morning and evening separately.

\subsubsection{The third diagnosis}

Systemic symptoms were significantly relieved, and the left breast pain was less than before. The tongue is red with thin white coating and wiry and rapid pulse. Dialectical analysis showed insufficiency of the kidney-yang and stagnation o $\mathrm{f}$ the circulation of vital energy and liver stagnation.

Prescription: Radix bupleuri12g; Scutellaria baicalensis 9g; Rhizoma sparganii 9g; Zedoary rhizome 9g; Astragalus membranaceus 20g; Poria cocos 15g; The rhizome of Chinese atractylode $15 \mathrm{~g}$; Nutgrass galingale rhizome 9g; Raw keel 20g; Raw oyster 20g; Fructus Aurantii Immaturus 15g; Lychee seed 15g; Cornu cervi degelatinatum 15g; Herba cistanche 15g; Gardenia 12g; Fructus Aurantii 6g; Rhizoma polygonati $15 \mathrm{~g}$

14 doses in total, and administer one dose a day in morning and evening separately.

\subsubsection{The fourth diagnosis}

Left breast pain is better than before, with occasional channeling pain. The pain is not obvious when touched. Symptoms showed that the tongue was red, the coating on the tongue was yellow, and the pulse was wiry.

Prescription: Peony bark 9g; White peony root 9g; Honey-fried licorice root $3 \mathrm{~g}$

14 doses, one dose a day, morning and evening separately.

\subsubsection{The fifth diagnosis}

Symptoms have been completely relieved. The left breast is soft without tenderness. Stop administration of the medicine.

\subsection{Case 4}

A 49-year-old male patient presented with bilateral breast enlargement and pain for three months. Breast ache is paroxysmal dull pain, ache is slight, appear when touching more. The patient had a 10 -year history of type 2 diabetes and a 6-year history of severe alcoholic cirrhosis, accompanied by hepatic encephalopathy, cholestasis, and ascites. Physical examination: bilateral breast symmetrically raised, areola staining, glandular tissue can be touched under the nipple, clear boundary, tough quality, gentle tenderness; tongue substance stasis purple, moss thin yellow greasy, pulse string. B-ultrasonography in the other hospital showed glandular echo with thickness of $10.3 \mathrm{~mm}$ in the right breast, and glandular echo with thickness of $10.6 \mathrm{~mm}$ in the left breast. The glandular structure was disorganized with uneven thickening of echo. Suggest male mammary gland development.

\subsubsection{The initial diagnosis}

Dialectical analysis showed that exogenous pathogenic factors can cause injury to the liver. The treatment can be made to soften and nourish the liver, regulate Qi and remove stasis. 
Prescription: Codonopsis pilosula 15g; Radix Ophiopogonis 9g; Radix rehmanniae recen 15g; The fruit of Chinese wolfberry 15g; Angelica sinensis 12g; Ligusticum wallichii 15g; Thunberg Fritillary Bulb 12g; The seed of Chinese dodder $10 \mathrm{~g}$; Radix scrophulariae $15 \mathrm{~g}$; the Root bark of the peony tree $9 \mathrm{~g}$; Raw oyster shell $20 \mathrm{~g}$; Prunella $15 \mathrm{~g}$

Take one dose a day in morning and evening (7 dosages).

\subsubsection{The further consultation}

Family consultation. The breast pain was slightly relieved and it was difficult to fall asleep at night. Tongue image photos showed dark tongue, visible tooth marks and thin yellow coating.

Dialectical analysis: Cortex albiziae 6g; Jujube kernel 6g; Poria cocos 15g; Atractylodes $15 \mathrm{~g}$

14 doses in total, and administer one dose a day in morning and evening separately.

1.4.3 The third diagnosis after two months

During this period, he was hospitalized in Beijing Ditan Hospital for surgery due to right inguinal hernia, so he stopped taking medication. At present, the indexes of liver function and blood sugar are good, and there is no obvious pain in the breast. Due to taking the traditional Chinese medicine prescription "Severe Liver Symptom No. 2" in Beijing Ditan Hospital, no further prescription has been given. Follow-up is currently under way.

\section{Discussion}

\subsection{Etiology and pathogenesis}

Ulcers experience collection-Mastoid carbuncle syndrome pointed out: "the male nipple is dominated by liver, breast is by kidney, to liver and kidney blood deficiency, kidney deficiency Jing, so swollen carbuncle." Chen Shigong discussed the etiology and pathogenesis of the disease in his book On Breast Carbuncle in Surgery, saying that "the breast segments of men are slightly different from those of women. Women damage the liver and stomach, men damage the liver and kidney, and the desire to cover the angry room is excessive. As a result, the liver is deficient in blood and dry, the kidney is deficient in essence and timid, the blood vessels cannot rise, the liver meridian cannot nourish and grow, and hence the swelling and pain". This conclusion has been inherited by most doctors in later generations ${ }^{[4]}$.

Compilation of Medical Records of Foreign Syndrome-Axillary and Ribs of Breast records: "a man's breast is a kidney, why also? Men with gas, women with blood as the first, foot Shaoyin kidney veins, bladder, its straight from the kidney through the diaphragm, into the lung, a little water Yang, straight through the three Yin above. Water does not contain wood, wood gas is not comfortable, true Yang can not reach. Tuberculosis in breast, qi depression... Although cloud liver disease, its origin in the kidney". Kidney storage essence, liver storage blood, blood for the mother and son of the dirty. The liver's function of blood storage and main catharsis depends on the warm support of kidney qi. If congenital endowment is insufficient, kidney qi is not sufficient; or old and infirmity, kidney deficiency; or a long illness and kidney, kidney loss, resulting in kidney deficiency of Yin and Yang so that kidney deficiency, kidney gas deficiency, blunt disorders. The two veins of Chong Ren start from the cell, Ren is the sea of Yin veins, and follow the abdomen, the upper Guan Yuan reaches the chest, Chong is the pulse of the sea of blood and goes up to the umbilical cord, and spreads to the chest. Imbalanced circulation of qi and blood in meridians leads to dysregulation, and meridians gather in milk collaterals, leading to paludism of milk ${ }^{[5]}$. The liver is the main regulator of qi. If the liver is derelict in its discharge, liver qi stagnation, qi stagnation and blood stasis can be caused, and then stagnation,dysfunction of the spleen in transport can be caused, and phlegm can be diagnosed with moisture, there will be qi stagnation, blood stasis, phlegm condensation in the nipple meridian, and the disease will occur as impassability of nipple meridian. Therefore, the deficiency of kidney qi, the disorder of flush-Ren, and the stagnation of liver qi are the root causes of the disease, and the loss of spleen health, qi stagnation and phlegm stasis are the signs of this disease. So the occurrence of men's breast development disease and liver and kidney dysfunction is closely related, the pathological changes of the viscera is mainly liver and kidney.

\subsection{Syndrome differentiation and treatment}

The etiology of gynecomastia is complex, and different etiologies are treated differently. Adolescent patients with primary breast development have a tendency to self-heal and generally return to normal within 6 months. Adult and elderly primary patients are not easy to heal and should be treated actively.

According to the clinical characteristics and the pulse pattern of tongue coating, paludula can be divided into the following four types.

\subsubsection{Liver stagnation and Qi stagnation, liver stagnation and fire}

This type is more common in young and middle-aged men, who were irritable and it will bring injury to the liver, 
resulting in liver stagnation, qi mechanism disorders, qi and blood stasis and affect a qi. Liver stagnation can cause harm to spleen, which give rise to dysfunction of the spleen in transport, and then lead to water metabolism disorders. Qi stagnation and blood stasis will be converted into fire, which will burn liver and kidney body fluid, and the fluid would be finally condensed into phlegm, resulting in spittoon, qi stagnation, blood stasis, spittoon condensation on the meridian disturbance. The main symptoms are lumps in the breast, slight pain, more normal skin color, which was accompanied by irritability, more anxiety after the disease, pain and mood are closely related. Treatment can be made to ease the liver and clear fire, reduce phlegm and resolve masses. Dan Zhi Xiaoyao San He two Erchen Soup can be chosen. Commonly used drugs are Bupleurum, Paeonia, Gardenia, green peel, tangerine peel, turmeric, angelica, peony, poria, atractylodes, licorice, mint and so on. Chaihu, Yu Jin disperse stagnated liver qi to relieve depression, so that liver qi strip up. Paeonia lactiflora acerbity is bitter and slightly cold, nourishing blood to collect Yin, soft liver is slow and urgent. Angelica is capable of nourishing the blood and promoting blood circulation. Angelica, peony are capable of tonifying the liver body and helping the liver. Liver disease is easy to spread the spleen, so Atractylostomy, tuckahoe, licorice spleen yiqi, can be used as the adjuvant. Peppermint through the liver through heat. Yanhuso with severe flank pain. Upset, sleepless night plus acacia skin, jujube kernel. Blood deficiency plus Salvia miltiorrhiza, making Radix Polygoni. Yin deficiency and asparagus, trichosanthus, and sophora root.

\subsubsection{Liver and kidney Yin deficiency type}

More commonly occured in middle-aged men, accompanied by waist and knee soft, dizziness and dizziness, spermatorrhea, five irritable heat, sleep less dream, red tongue less moss, pulse number and other symptoms. The liver stores blood, the kidney stores essence, the fine blood interchanges and the homology, therefore also calls the liver and kidney homology. Liver Yin and kidney Yin breed and fill each other, full with full, decline with failure. Treatment should nourish Yin phlegm, soft and firm sanjie. Often choose ripe rehmannia rehmannii, dogwood, yam, alisma alisma, tuckahoe, peony bark, angelica, white peony, fritillaria chuan. Ripe rehmannia glutinosa nourishes Yin and tonifies kidney, filling lean pulp. The dogwood can nourish the liver and kidney, and can astringent essence. The yam can nourish the spleen and Yin, and can also consolidate the kidney. Rehmannia rehmannii, dogwood and Chinese yam are used together to supplement the liver, kidney and spleen with three Yin. Alisma alisma moistens, but also can reduce the ripe Rehmannia gluttony. Poria cocos moistens and moistens, helping the healthy function of yam. Peony skin is clear to relieve deficiency heat, white peony root nourishing the liver soften liver.

\subsubsection{Kidney-Yang deficiency type}

This one is seen more among seniors with mastauxy, which showed no symptoms of pain or the the slight pain is felt. Instead, there were symptoms of soreness and weakness of waist and knees with fatigue and greasy coating as well as weal pulse. Elderly males were prone to kidney Yang deficiency. Kidney Yang is the source of Yang Qi, aged and weak, or a long illness of kidney wer caused by the deficiency of Yin and Yang of the kidney, which failed to contain liver Yang, as a result, the gas is not ventilated with drainage derelicacy, so phlegm wet stop gather, rather it will gather in the nipple meridian, which was the cause of the disease. The treatment can be made with use of Epimedium, Xianmao, Weilingxian, Atractylodes, Rehmannia rehmanniae, antler cream. Xianmao, WeiLingXian, YuYangEpime has the effect of tonifying the kidney and warming the Yang, Bupleurum soothens the liver and regulates Qi, Rehmannia rehmanniae, deer horn cream warming the Yang and reducing phlegm.

\subsubsection{Liver injury caused by exogenous pathogens}

Most of them are caused by exogenous dampness-heat virus, or by long-term use of the products that hurt the liver and spleen, which is more commonly seen in liver diseases, or by long-term oral drugs. Exogenous pathogenic factors lead to deficiency of liver and blood, deficiency of liver Yin, loss of liver tenderness and stagnation of liver qi. Then it will further cause the spleen deficiency, unhealthy transport of spleen, endogenous phlegm turbidity, as a consequence, the stagnation of Qi and blood stasis will occur. Clinical common breast development is in bilateral way, breast swelling pain, the breasts may have lumps accompanied by pain in the right side, mouth bitter and sticky, fatigue, poor food intake, loose stools, purple tongue silt, thin yellow greasy coating, fine pulse. The treatment is made by using decocting and subtracting to soft liver nourishing the liver, regulate qi and disperse knot, commonly used Dangshen, Ophiopogon ophiopogonis, raw rehmannia glutinosa, medlar, dodder, angelica, chuanxiong, fritillaria thunbergii, peony bark, Radix Scrophulariae, oyster, shanci mushroom, prunella prunerae and so on.

\section{Summary}

Chinese medicine believes that the male nipple belongs to liver, breast belongs to kidney, if emotion is not adjusted, liver stagnation Qi will occur;it is likely to be caused by old age liver and kidney deficiency or lack of innate endowments 
and impulse and Ren disorder syndrome. Exogenous evil injury to the liver, liver loss and catharsis, can lead to the loss of meridians, Qi and blood is not smooth, thus blood stasis and cuspidor block the meridians and become male breast disease. Therefore, this disease is based on liver and kidney injury, and is marked by Qi stagnation, cuspidor coagulation and blood stasis. The treatment is based on syndrome differentiation of liver and kidney, following the principle of warming Yang and tonifying kidney, dredging liver and promoting blood circulation, or dredging liver and clearing fire, dispersing Jie and resolving phlegm. The treatment of gynecomastia based on syndrome differentiation of liver and kidney can not only adjust the whole body of the patient, but also treat the symptoms according to syndrome differentiation, which can be described as specimen treatment.Modern pharmacological studies also show that the traditional Chinese medicine of warming Yang and tonifying kidney, such as Xianling Spleen, has the effect of sex hormones. Salvia miltiorrhiza and Yujin can improve the blood circulation of the whole body and liver, contribute to the metabolism of hormones in the body, and eliminate the hyperemia and edema of breast tissue and the proliferation of fibrous connective tissue. By prolonging the thrombosis time, shortening the thrombosis length and reducing the weight of the thrombosis, the anti-thrombosis drugs can also improve the characteristics of hemorheology and reduce the blood viscosity.

The observation shows that it is better to treat gynecomastia according to the syndrome differentiation of liver and kidney. In addition, on the basis of drug therapy combined with psychological counseling, relieve the patient's ideological concerns, help patients recover as soon as possible.

\section{References}

[1] Wu Jieping, Qiu Fazu, Wu Mengchao, Wu Zaide. Huang Jiasi Surgery. 7th Edition (Volume II). Beijing: People's Medical Publishing House; 2008.

[2] Xin Zhifang. Management of gynecomastia. Chinese Journal of Breast Diseases. 2009; 3(4): 412-418.

[3] Lin Yi, Tang Hanjun. Modern Chinese Medicine Mammatology. Beijing: People's Medical Publishing House; 2003.

[4] Qian Xiaoqiang. Treatment of 30 cases of gynecomastia with Xiaofu decoction. Journal of Traditional Chinese Medicine. $1999 ; 15(6): 38$. 